\title{
Coronary Artery Bypass Graft Surgery in Tanzania: 5 Years of Experience at Jakaya Kikwete Cardiac Institute
}

Mohamed Yakubu Janabi

$\mathrm{JKCl}$

Evarist Nyawawa

$\mathrm{JKCl}$

Bashir Nyangasa

$\mathrm{JKCl}$

William Ramadhani Ramadhan

$\mathrm{JKCl}$

Ramadhani Hassan Hamis

$\mathrm{JKCl}$

Hassanali Hussein

$\mathrm{JKCl}$

Godwin Sharau

$\mathrm{JKCl}$

Majani Naizibijwa

$\mathrm{JKCl}$

Khuboja Sulende

$\mathrm{JKCl}$

Vivienne Aiyana Mlawi ( $\sim$ vmlawi9@gmail.com )

Jakaya Kikwete Cardiac Institute https://orcid.org/0000-0002-4152-5239

Tulizo Shemu

$\mathrm{JKCl}$

Smita Bhalia

$\mathrm{JKCl}$

Peter Kisenge

$\mathrm{JKCl}$

Delilah Kimambo

$\mathrm{JKCl}$

Engerasia Kifai

$\mathrm{JKCl}$

Waane Tatizo 
$\mathrm{JKCl}$

\section{Henry Mayala}

$\mathrm{JKCl}$

Khuzema Khanbhai

$\mathrm{JKCl}$

\section{Boniface Waandwi}

$\mathrm{JKCl}$

\section{Angela Muhozya}

$\mathrm{JKCl}$

\section{Research article}

Keywords: Cardiac Surgery, sub-Saharan Africa (SSA), Jakaya Kikwete Cardiac Institute (JKCI), Cardiovascular Disease (CVD), Coronary Artery Bypass Graft (CABG).

Posted Date: October 1st, 2020

DOI: https://doi.org/10.21203/rs.3.rs-73049/v1

License: (c) (7) This work is licensed under a Creative Commons Attribution 4.0 International License. Read Full License 


\section{Abstract}

Cardiac surgery is not widely available in most developing countries, and most patients have no choice but to live in morbid conditions and managed conservatively or the few who are referred abroad for surgical procedures costs the respective countries millions of hard earned foreign currency. The World Health Organization projects that over the next ten years the continent of Africa will experience the largest increase in death rates from cardiovascular disease. The Jakaya Kikwete Cardiac Institute $(\mathrm{JKCl})$ is a government owned National Specialized and Teaching Hospital that serves patients from all the regions of the United Republic of Tanzania with a population of nearly $60,000,000$ people and also serves beyond the borders (Rwanda, Burundi, DR Congo, South Sudan, Comoro, Malawi and Zambia) for advanced cardiovascular medical, intervention, vascular and open heart surgery, the Institute was established in 2015.

Methods: Here we report all patients who underwent coronary artery bypass surgery grafting only performed at the Centre since its inauguration in 2015- till 2019. Data were collected for basic demography, diagnosis, investigations, clinical and surgical outcome parameters.

Results: A total of 85 patients with heart diseases and underwent coronary artery bypass surgery grafting (CABG) are analysed in this study. There were 64 (75\%) male and $21(25 \%)$ female patients. Their age ranged from 41-85 years old with almost half $42(49 \%)$ of the cohort being between the age between 61-70 years old. Most of the patients had two or more grafts and an internal mammary artery graft was used over $80 \%$ of the procedures. The overall 30 -day mortality was $7.1 \%$, incidence of stroke $0.2 \%$, duration of mechanical ventilation was an average of 9.98 hours and intensive care unit (ICU) stay post CABG was an average of 6.48 days and final discharge from the centre ranged from 10-16 days.

Conclusion: This study has demonstrated that coronary artery bypass surgery grafting in low/middle income country is safe and feasible. A sustainable program demands highest level of governmental support as seen in this case, and a dedicated multidisciplinary team with profound know how in cardiac pathologies. Furthermore, a need for good local data to know the prevalence of coronary disease is mandatory to determine the magnitude of coronary artery disease in each country.

\section{Introduction}

Africa, home to 54 low and middle-income countries, will have the world's largest increase in NCD deaths over the next decade. Although communicable diseases such as malaria, TB and HIV/AIDS and other conditions still predominate in sub-Saharan Africa, WHO projects that, by 2030, NCDs will become the leading cause of death. This would impose a significant burden on the continent, whose population will double within the next generation. ${ }^{1}$

The Jakaya Kikwete Cardiac Institute $(\mathrm{JKCl})$ is a government owned National Heart Diseases Specialized and Training Centre established in 2015, that serves patients from all regions of the United Republic of Tanzania and beyond for advanced cardiovascular treatment. The Institute has 150 bed capacities for 
inpatients. In the 5 years it has admitted over 15,461 cardiac patients, attended 323,590 outpatients, performed 4207 interventions and undertook 2141 open heart and vascular surgeries. The first bypass surgery was performed in August 2016. This work aimed at assessing the first 5 years of this program on only coronary artery bypass surgery grafting $(C A B G)$ other surgeries are reported elsewhere.

\section{Methods}

\section{Study design}

We carried out a cross-sectional descriptive study in the cardiovascular surgical unit of $\mathrm{JKCl}$ from 2015 to 2019. We reviewed the medical files of all patients who underwent coronary artery bypass surgery grafting.We collected information on their; age, sex, city of origin, past medical history, symptoms and signs at presentation, nature of heart disease, surgical procedure, post-procedure complications, and outcome within 30 days.

\section{Procedure}

All surgeries used a standard operating approach involving right aorta-atrial cardiopulmonary bypass using heart lung machine. Following induction of anaesthesia, a median sternotomy approach was used. The conduits were harvested as usual and cardiopulmonary bypass was established using right atrial and aortic cannula, following heparin infusion. Myocardial protection was achieved with moderate hypothermia (28-32 C). Distal coronary anastomoses were performed on a still heart. Post completion of the grafting on coronaries and rewarming, the aortic cross-clamp was removed and the proximal ends of the vein grafts were anastomosed to the aorta under a partially occluding clamp. In selected patients, a single-clamp technique was used for both distal and proximal anastomoses. Once the reperfusion was established through grafts, the heart was gradually weaned from the heart lung machine and subsequent chest closure was done.

\section{Statistical analysis}

Data were analysed using the software EPI Info Version 3.1 (Centres for Disease Control and Prevention, Atlanta, Georgia, USA). We present quantitative data as frequencies and percentages. And continuous variables are presented as mean \pm standard deviation (SD).

\section{Results}

From 2015 to the end of 2019 a total of 85 patients with coronary artery disease and had indication for coronary artery bypass surgery grafting were operated. Demographically there were 64 (75\%) male vs. 21 (25\%) female patients. Their age ranged from 41-85 years old and almost half of the patients were between the ages of 61-70 years old $42(49 \%)$. Study patients had involvement of two or more grafts while internal mammary artery graft was used in over $80 \%$ of the procedures performed. The overall $30-$ day mortality was $7.1 \%$; incidence of stroke $0.2 \%$, duration of mechanical ventilation was an average of 
9.98 hours and ICU stay post CABG at 6.48 days and final discharge from the centre ranged from 1016 days.

Table 1 demonstrates the pre-operative demographic characteristics of the study subjects, Main risk factors noted were obesity, hypertension, diabetes mellitus and dyslipidaemia with low rates of smoking among the study patients. In terms of outcomes Table 2 shows, overall mortality for the cohort was $8 \%$. The 30 days' mortality is shown in Table 3.

\section{Discussion}

While, communicable, maternal, neonatal, and nutritional diseases decline, particularly from 2005 onwards. The growth in population size and life style change in sub-Sahara Africa was the key driver of the rise of NCD burden from the same time period. ${ }^{1}$ Zack R et al showed in a study conducted in Tanzania that hypertension prevalence was 37\%. ${ }^{1,2}$ Major risk factors for hypertension in Dar es Salaam were; overweight, obesity, inadequate physical activity, and limited access to quality medical care. In North Africa, NCDs are already responsible for more than three-quarters of all deaths, and nearly half the population in this region already suffers from hypertension, a well-established precursor to NCDs such as heart diseases, according to the WHO's non-communicable diseases country profiles for $2014 .{ }^{1,2}$ In Algeria, Egypt, Libya and Morocco, for example, more than 75\% of all deaths in 2012 were due to NCDs. Of immediate concern to public health planners is that $30 \%$ of all people in Africa have high blood pressure and will most likely suffer from coronary heart diseases, stroke, renal or visual impairment or other related conditions. ${ }^{1,2,3}$

However, despite this rise in Africa of NCDs as shown above, a large gap exists for access to quality cardiovascular services and health education among the population. In developed countries such as North America, Australia, and Europe, the average number of cardiac surgical cases performed was 860 per 1 million people as of 2008.3,4 On the other spectrum, in developing countries such as South America, Russia, Asia, and Africa, the average number of cardiac surgical cases performed was 60 per 1 million people. Therefore, $93 \%$ of people who require cardiac surgery living in developing countries, an estimated 4.5 billion people total, do not have access to this advanced treatment. ${ }^{1,4,5,6}$

Here we report for the first time prospectively acquired outcome data from $\mathrm{JKCl}$ in Tanzania a lowermedium income country. The World Bank has recategorised Tanzania as a lower-middle income country after the country made economic reforms, including making consistent plans and taking hard decisions aimed at improving its economic development. From 2015 to the end of 2019 a total of 85 patients with coronary artery disease who had indication for coronary artery bypass surgery grafting were operated. A sustainable program such as this which just celebrated its 5th birthday, demands the highest level of governmental support and a dedicated multidisciplinary team with profound know how in cardiac pathologies lead to the success observed in this initial five years. The continuity of the project requires further and sustained re-enforcement of technical platform, increase the human resource capacities, and subsidise surgery through universal health coverage due to the low uptake encountered. As of 2011 , the 
estimated cost of open heart surgery in developing countries reported was between $\$ 6,230$ and $\$ 11,200$ USD depending on the procedure, which is much less than seeking surgical care overseas $(\$ 20,000-$ $\$ 40,000$ USD per traveller). ${ }^{7,8}$ Procedure expenses could be further reduced by $\sim \$ 1,000$ USD if consumables and cardiac prostheses costs were reduced and manufactured locally. Lack of local cardiac centres within African countries contributes to millions of dollars lost annually to international referrals. ${ }^{9}$

To worsen the situation most developing countries sub-Sahara Africa among them lack an efficient health insurance system, affordability is one of the most critical aspects of local cardiac centres. Therefore, the patient's bill must reflect an amount for the centre to break even. Patients of lower socioeconomic status might need sponsorship by the government, NGO or private donor. ${ }^{10,11}$

Therefore, initiating local cardiac programs will ultimately benefit patients, benefactors, and the country's economy as seen in our case of Tanzania demonstrated here where $95 \%$ of the cardiac surgeries beside CABG are performed within the country saving billions of hard earned forex used before 2015 to refer patients abroad. Cardiac surgery programme here could be considered as good when compared to that reported in the literature in other low income countries.

\section{Conclusion}

The need for cardiac surgical centres in developing countries and underserved areas of the world is critical to help ease this gap in care. The enormity of this issue necessitates a collaborative effort from international partners, NGOs, local governments, and private benefactors to facilitate adequate cardiac care for the African's population and to overcome the many challenges associated with establishing cardiac programs.

\section{Abbreviations}

JKCl: Jakaya Kikwete Cardiac Institute

CABG: Coronary artery bypass graft

USD: United state dollar

NCD: Non communicable disease

NGO: Non governmental organization

ICU: Intensive Care unit

HIV: Human immunodeficiency virus

WHO: World health organization 


\section{Declarations}

\section{Acknowledgement}

Sincere thanks to all JKCl staff and patients for kind cooperation during data collection.

\section{Consent for Publication}

Not applicable

\section{Availability of Supporting data}

The raw data supporting the findings of this paper will be available to editorial committee at any point if required

\section{Funding}

Self funded

\section{Authors contributions}

JB and AM: Conceived the study

EN, BN, RW, RRH, HH, BW and GS: Performed surgeries

VM,SB and KK: Post operative care and follow up

KS, TS, DK and EF: Performed detailed Echocardiography

PK, WT, HM and MN: Contributed to analyse data and prepare final version of manuscript

\section{Competing Interest}

All authors declare no competing interest.

\section{Ethical statement}

This work was approved by the institutional review board of the Jakaya Kikwete Cardiac Institute. We carried out this work in accordance with the declarations of Helsinki. We report this work according to the Standard for Reporting Observational Study (STROBE) checklist.

\section{References}

1. Determinants Of High Blood Pressure And Barriers To Diagnosis And Treatment In Dar Es Salaam, Tanzania Rachel M. Zack, Kahema Irema, Patrick Kazonda, Germana H. Leyna, Enju Liu,Donna 
Spiegelman, Wafaie Fawzl, Marina Njelekela, Japhet Killewo, Goodarz Danaei, J Hypertens. 2016 Dec; 34(12): 2353-2364.

2. GBD 2017 Causes of Death Collaborators. Global, regional, and national age-sex-specific mortality for 282 causes of death in 195 countries and territories, 1980-2017: a systematic analysis for the Global Burden of Disease Study 2017. Lancet. 2018; 392: 1736-1788.

3. Dalal S, Beunza J, Volmink J et al. Non-communicable diseases in sub-Saharan Africa: what we know now. Int J Epidemiology. 2011; 40: 885-901

4. Touze JE. Cardiovascular diseases and the epidemiological transition in tropical regions. Med Trop (Mars) 2007; 67:541-2.

5. Diarra MB, Ba HO, Sanogo KM, et al. The cost of evacuations for cardiovascular diseases and the needs for surgical and interventional treatment in Mali. Cardiol Trop 2006; 32:66-8.

6. Moore AG. Rheumatic Heart Disease. In: Murphy JG, Lloyd MA. Editors. Mayo Clinic Cardiology, 3rd edition. Rochester: Mayo Clinic Scientific Press, 2007:549-54.

7. Kingue S, Ngodjoum N, Muna W, et al. The Cameroon experience of Cardiac surgery: a report of 83 cases operated from 1985 - 1990. Cardiol Trop 1994; 20:5-11.

8. Ngatchou W, Lemogoum D, Ménanga AP, et al. Cardiac surgery in Cameroon. Results at one year of the pilot phase. Rev Med Brux 2011; 32:14-7.

9. Marijon E, Ou P, Celermajer DS, et al. Prevalence of rheumatic heart disease detected by echocardiographic screening. N Engl J Med 2007; 357:470-6.

10. Anyanwu $\mathrm{CH}$, Ihenacho $\mathrm{HN}$, Okoroma EO, et al. Initial experience in open heart surgery in Nigeria. Trop Cardiol 1982; 8:123-7.

11. Metras D, Coulibaly A, Ouattara K, et al. Cardiac surgery at the Institute of cardiology of Abidjan 1978-1983. Study of 728 surgical operations including 501 open hearts. Cardiol Trop 1984; 81-7.

12. Moore AG. Rheumatic Heart Disease. In: Murphy JG, Lloyd MA. Editors. Mayo Clinic Cardiology, 3rd edition. Rochester: Mayo Clinic Scientific Press, 2007:549-54.

\section{Tables}

Table 1. Sociodemographic characteristics 


\begin{tabular}{|ll|}
\hline Variable & No. (\%) \\
\hline Age Mean (SD) & \\
\hline & 0 \\
\hline $41-50$ years & \\
\hline $51-60$ & $5(6 \%)$ \\
\hline $61-70$ & $26(31 \%)$ \\
\hline $71-80$ & $42(49 \%)$ \\
\hline$>80$ & $10(12 \%)$ \\
\hline Sex & $2(2 \%)$ \\
\hline Male (\%) & $64(75 \%)$ \\
\hline Female (\%) & $21(25 \%)$ \\
\hline
\end{tabular}

Table 2. Risk factors

\begin{tabular}{|ll|}
\hline Variable & No. \\
\hline Diabetes Mellitus & $12(23.5)$ \\
\hline Hypertension & $51(79.8)$ \\
\hline Hypertension/ Diabetes Mellitus & 32 \\
\hline Weight mean (SD) in kg & $75.41 \pm 14.5$ \\
\hline BMl & $28.75 \pm 5.3$ \\
\hline Smoking & $5(5.8 \%)$ \\
\hline Alcohol & $22(25 \%)$ \\
\hline Combined All & $7(8 \%)$ \\
\hline Total cholesterol (mmol/l) & $5.02 \pm 1.5$ \\
\hline Triglyceride (mmol/l) & $1.7 \pm 0.88$ \\
\hline HDL(mmol/l) & $1.08 \pm 0.34$ \\
\hline LDL(mmol/l) & $3.3 \pm 1.3$ \\
\hline
\end{tabular}


Table 3. Outcome of bypass surgery within $\mathbf{3 0}$ days

\begin{tabular}{|c|c|c|c|}
\hline Characteristics & & No of patients & $(\%)$ \\
\hline \multicolumn{4}{|l|}{ Overall mortality } \\
\hline & & 6 & 7.1 \\
\hline \multirow[t]{2}{*}{7 days survival } & Alive & 79 & 92.9 \\
\hline & Dead & 6 & 7.1 \\
\hline \multirow[t]{2}{*}{1 months follow up } & Alive & 79 & 92.9 \\
\hline & Dead & 0 & 7.1 \\
\hline
\end{tabular}

